\title{
Efficient Computation of the Shapley Value for Centrality in Networks
}

\author{
Karthik .V. Aadithya ${ }^{1}$ Balaraman Ravindran ${ }^{2}$ Tomasz P. Michalak ${ }^{3}$ \\ Nicholas R. Jennings ${ }^{3}$ \\ ${ }^{1}$ The University of California, Berkeley, USA \\ aadithyadeecs.berkeley.edu \\ ${ }^{2}$ Indian Institute of Technology Madras, India \\ ravidcse.iitm.ac.in \\ ${ }^{3}$ School of Electronics and Computer Science, University of Southampton, UK \\ $\{$ tpm, nrj\}@ecs.soton.ac.uk
}

\begin{abstract}
The Shapley Value is arguably the most important normative solution concept in coalitional games. One of its applications is in the domain of networks, where the Shapley Value is used to measure the relative importance of individual nodes. This measure, which is called node centrality, is of paramount significance in many real-world application domains including social and organisational networks, biological networks, communication networks and the internet. Whereas computational aspects of the Shapley Value have been analyzed in the context of conventional coalitional games, this paper presents the first such study of the Shapley Value for network centrality. Our results demonstrate that this particular application of the Shapley Value presents unique opportunities for efficiency gains, which we exploit to develop exact analytical formulas for Shapley Value based centrality computation in both weighted and unweighted networks. These formulas not only yield efficient (polynomial time) and error-free algorithms for computing node centralities, but their surprisingly simple closed form expressions also offer intuition into why certain nodes are relatively more important to a network.
\end{abstract}

\section{Introduction}

The Shapley Value (SV) is a fundamental normative solution concept in coalitional games. Given a scenario where agents are allowed to realize collective payoffs through mutual co-operation, the SV postulates a fair method to evaluate each agent's individual contribution. One of the many applications of the SV is in the domain of networks, where it is used to measure the importance of individual nodes, which is known as game theoretic network centrality [1,2]. Although centrality plays a key role in many reallife network applications, efficient algorithms for its measurement via the SV remain unknown.

We now introduce the concept of "centrality". In the networks context, it is often paramount to determine which nodes and edges are more critical than others. Classic examples include identifying the most important highways in a road network, the most influential people in a social network or the most critical functional entities in a protein network. As a result, the concept of centrality, which aims to quantify the importance of individual nodes/edges in a network, has been extensively studied in network analysis. Such a conventional centrality metric, however, suffers from the following drawbacks: 
1. By considering only the failure of individual nodes, it completely ignores real-world situations where multiple nodes can fail simultaneously. For example, if the network is so designed that no single node's failure carries any consequence, but the failure of certain specific pairs of nodes can bring down the entire network, the above centrality metric would fail to give a higher centrality score to the nodes belonging to these critical pairs.

2. Because each node is treated separately, the hidden assumption is that node failures occur independently of each other. As a result, real-world phenomena such as cascading node failures, that have been known to precipitate widespread disruption in a very short time [3], are outside the scope of this centrality analysis.

In short, conventional centrality measures fail to recognize that in many network applications, it is not sufficient to merely understand the relative importance of nodes as stand-alone entities. Rather, the key requirement is to understand the importance of each node in terms of its utility when combined with other nodes [4]. For instance, in the above infrastructure network, an ideal centrality measure would assign a score to a node $v$ based on the failure probabilities (and consequences thereof) of every subset of nodes containing $v$, rather than just failure of the single node $v$. This approach would automatically allow the ideal centrality measure to give due consideration to real-world failure patterns such as cascading failures and simultaneous multiple node failures. On the other hand, this flexibility, which comes from the ability to take into account the contributions of all possible combinations of nodes (rather than just one node at a time), is absent in conventional centrality measures, which is a crucial limitation in many applications.

Game theoretic network centrality $[1,2]$ has been proposed as a framework that would address this limitation. Given the network to be analysed, the idea is to define a cooperative game where the agents (players) are the nodes of the network. Then the SV of each agent (node) in this game is interpreted as a centrality measure because it represents the average marginal contribution made by each node to every possible combination of the other nodes. This paradigm of SV-based network centrality thus confers a high degree of flexibility (which was completely lacking in traditional centrality metrics) to model real-world network phenomena. Indeed, this new paradigm has already been proved to be more useful than traditional centrality measures for certain real-life network applications $[1,5]$.

From a computational perspective, however, evaluating game theoretic network centrality using the original SV formula involves an analysis of the marginal contribution of every node (i.e. player) to every coalition. Thus, given a network $G(V, E)$, a direct application of the SV formula involves considering $O\left(2^{|V(G)|}\right)$ coalitions. Such an exponential computation is clearly prohibitive for bigger networks (of, e.g, 100 or 1000 nodes). For such networks, the only feasible approach currently outlined in the literature is Monte-Carlo sampling, which is not only inexact, but also very time-consuming.

The above problem of exponential complexity in the number of agents is a fundamental challenge associated with computing the SV. As a result, for conventional coalitional games, this issue has received considerable attention in the literature. As an alternative to the straightforward (but exponential) listing of all possible coalitions, some authors $[6,7]$ have proposed more efficient representations for coalitional games. In addi- 
tion to being concise for many games, these representations are expressly designed to possess desirable computational properties, including efficient SV computation. Thus, the choice of representation has been the foremost consideration for efficient SV computation in the context of conventional coalitional games.

The networks domain, by contrast, poses a very different set of challenges:

1. Unlike conventional coalitional games, conciseness is usually not an issue in the networks context. This is because the games that aim to capture network centrality notions are completely specified by (a) the underlying network compactly represented as a graph, and (b) a concise closed-form characteristic function expression for evaluating coalition values (please see next section for an example). Rather, the issue here is that the exact specification for the characteristic function is dictated not by computational considerations, but by the real-world application of game theoretic network centrality.

2. Because the games in this paper are designed to reflect network centrality, the characteristic function definition often depends highly non-trivially on the underlying graph structure.

Therefore, the challenge we face in this paper is to efficiently compute the SV, given a network and a game defined over it, where coalition values for this game are given by a closed-form expression that depends non-trivially on the network. The key question here is how to take advantage of (a) the network structure, and (b) the functional form for the coalition values, so as to compute SVs efficiently, i.e, without the need to enumerate all possible coalitions.

Against this background:

[1] Our key contribution in this paper is to demonstrate that it is possible to exactly and efficiently compute SV-based network centralities of practical interest defined on large networks which exceed thousands of nodes! By contrast, the only previously known method that scaled to such large networks was Monte-Carlo simulation, which was neither exact nor particularly efficient.

[2] For four different measures of network centrality, we develop exact closed-form formulas for the SVs. We present pseudo-codes of linear and polynomial time algorithms to implement these formulas.

[3] We develop a closed-form polynomial time computable SV approximation for a fifth measure of centrality defined on weighted networks.

[4] We test our algorithms on two real-life examples, (a) an infrastructure network representing the topology of the Western States Power Grid, and (b) a collaboration network from the field of astrophysics. The results show that the algorithms proposed in this paper are not only accurate but also deliver significant speedups (upto 550x for the 16000+ node scientific collaboration network) over Monte-Carlo simulation.

The remainder of the paper is organized as follows. Section 2 presents an example of how a coalitional game may be used to capture the notion of network centrality. Section 3 analyses four types of centrality-related coalitional games and presents polynomial time SV algorithms for all of them. Conclusions follow. 


\section{SV as a Centrality Measure}

As mentioned in the introduction, the paradigm of game theoretic network centrality based on the SV has been proposed in [1,2] and further explored in [5]. This section presents an example to illustrate the advantages of this paradigm over conventional centrality measures.

Consider the notion of "closeness centrality" of a node in a graph $G(V, E)$, which is traditionally defined as the reciprocal of the average distance of that node from other (reachable) nodes in the graph [8]. This definition captures the intuitive idea that a node "in close proximity to many other nodes" is more valuable by virtue of its central location, and hence should be assigned a higher centrality score.

The above measure, however, fails to recognize the importance of combinations of nodes. For example, consider a typical real-world application of closeness centrality: that of disseminating a piece of information to all nodes in the network. At any time point $t$ in the dissemination process, define the random variable $C_{t}$ to be the subset of nodes most actively involved in propagating the information. In this situation, a new node added to $C_{t}$ would make maximum contribution to the diffusion of information only if it is "in close proximity to nodes that are not currently in close proximity to any node in $C_{t}$ ". Thus, while conventional closeness centrality only takes into account average proximity to all other nodes, the actual importance of a node in the real-world application is based on a very different measure: proximity to nodes that are not in close proximity to the random variable $C_{t}$.

We now show how coalitional game theory can be used to construct a centrality measure that faithfully models the above real-world importance of a node. Let $C$ be any subset of nodes from the given network $G(V, E)$. Then, for every such $C$, assign a value $\nu(C)$ given by

$$
\nu(C)=\sum_{v \in V(G)} \frac{1}{1+\min \{d(u, v) \mid u \in C\}}
$$

where $d(u, v)$ is the distance between nodes $u$ and $v$ (measured as the shortest path length between $u$ and $v$ in graph $G$ ).

The map $\nu$ defined above captures a fundamental centrality notion: that the intrinsic value of a subset of nodes $C$ in the context of a real-world application (such as information dissemination) is proportional to the overall proximity of the nodes in $C$ to the other nodes in the network. In effect, the map $\nu$ carries the original definition of closeness centrality to a global level, where a measure of importance is assigned to every possible combination of nodes.

The map $\nu$ above is therefore a characteristic function for a coalitional game, where each vertex of the network is viewed as an agent playing the game. It follows that if a node $v$ has a high $\mathrm{SV}$ in this game, it is likely that $v$ would "contribute more" to an arbitrary randomly chosen coalition of nodes $C$ in terms of increasing the proximity of $C$ to other nodes on the network. Thus, computing the SVs of this game yields a centrality score for each vertex that is a much-improved characterization of closeness centrality.

The only difficulty in adopting such a game-theoretically inspired centrality measure is the previously mentioned problem of exponential complexity in the number of agents. 
In the next section, we show how to overcome this difficulty and compute the SV for many centrality applications (including the above formulation) in time polynomial in the size of the network.

\section{Algorithms for SV-based network centrality}

In this section, we present 5 characteristic function formulations $\nu(C)$, each designed for a different real-world application. While each formulation captures a different $f l a-$ vor of centrality, they all embrace one fundamental centrality idea: that the definition for $\nu(C)$ must somehow quantify the sphere of influence of the coalition $C$ over the other nodes. For instance, in our first game formulation, we start with the simplest possible idea that the sphere of influence of a coalition of nodes $C$ is the set of all nodes immediately reachable (within one hop) from $C$. Subsequent games further generalize this notion of sphere of influence. For example, the second formulation specifies a more sophisticated sphere of influence: one that includes only those nodes which are immediately reachable in at least $k$ different ways from $C$. The other three formulations extend the notion of sphere of influence to weighted graphs. The third game, for instance, defines sphere of influence as the set of all nodes within a cutoff distance of $C$ (as measured by shortest path lengths on the weighted graph). The fourth and final formulation is an extreme generalization: it allows the sphere of influence of $C$ to be specified by an arbitrary function $f($.$) of the distance between C$ and the other nodes.

Throughout this section, we assume the reader is familiar with concepts of graph theory, including weighted and unweighted graphs, vertex degrees, neighboring vertices and shortest paths. We do not define these concepts here but suggest the references $[9,10]$. The terms "network" and "graph" are used interchangeably in this paper, as are the terms "node" and "vertex". All the weighted graphs considered in this paper are positive weighted. We do not use digraphs in this paper, so all graphs are assumed to be undirected.

We also assume familiarity with the concepts of co-operative game theory, including the definition of coalitional games in characteristic function form and the Shapley Value. We do not define these concepts here but suggest the references [11,12].

We now set the notation for a general coalitional game played on a network. Given a graph $G(V, E)$ with vertex set $V$ and edge set $E$, we use $G$ to define a coalitional game $g(V(G), \nu)$ with set of agents $V(G)$ and characteristic function $\nu$. Here the agents of the coalitional game are the vertices of the graph $G$. Thus a coalition of agents $C$ is simply any subset of $V(G)$. The characteristic function $\nu: 2^{V(G)} \rightarrow \mathbb{R}$ can be any function that depends on the graph $G$ as long as it satisfies the condition $\nu(\emptyset)=0$. We use the phrase "value of coalition $C$ " to informally refer to $\nu(C)$.

\subsection{Game 1: $\nu_{1}(C)=$ \#agents at-most 1 degree away}

Given an unweighted, undirected network $G(V, E)$. We first define "fringe" of a subset $C \subseteq V(G)$ as the set $\{v \in V(G): v \in C$ (or) $\exists u \in C$ such that $(u, v) \in E(G)\}$, i.e, the fringe of a coalition includes all nodes reachable from the coalition in at most one hop.

Based on the fringe, we define the coalitional game $g_{1}\left(V(G), \nu_{1}\right)$ with respect to the network $G(V, E)$ by the characteristic function $\nu_{1}: 2^{V(G)} \rightarrow \mathbb{R}$ given by 


$$
\nu_{1}(C)= \begin{cases}0 & \text { if } C=\emptyset \\ \operatorname{size}(\text { fringe }(C)) & \text { else }\end{cases}
$$

This coalitional game has been extensively discussed in [1], where the authors motivate the game by arguing that the SVs of nodes in this game constitute a centrality metric that is superior to degree centrality for some applications. It is therefore desired to compute the SVs of all nodes for this game. We shall now present an exact formula for this computation rather than obtaining it through Monte-Carlo simulation as was done in [1].

To evaluate the SV of node $v_{i}$, consider all possible permutations of the nodes in which $v_{i}$ would make a positive marginal contribution to the coalition of nodes occurring before itself. Let the set of nodes occurring before node $v_{i}$ in a random permutation of nodes be denoted $C_{i}$. Let the neighbors of node $v_{i}$ in the graph $G(V, E)$ be denoted $N_{G}\left(v_{i}\right)$ and the degree of node $v_{i}$ be denoted $\operatorname{deg}_{G}\left(v_{i}\right)$.

The key question to ask is: what is the necessary and sufficient condition for node $v_{i}$ to marginally contribute node $v_{j} \in N_{G}\left(v_{i}\right) \cup\left\{v_{i}\right\}$ to fringe $\left(C_{i}\right)$ ? Clearly this happens if and only if neither $v_{j}$ nor any of its neighbors are present in $C_{i}$. Formally $\left(N_{G}\left(v_{j}\right) \cup\right.$ $\left.\left\{v_{j}\right\}\right) \cap C_{i}=\emptyset$.

Given that permutations are chosen uniformly at random for computing the SV, combinatorial arguments can be used to show that the above condition is satisfied with probability $\frac{1}{1+\operatorname{deg}_{G}\left(v_{j}\right)}$. Denote by $B_{v_{i}, v_{j}}$ the Bernoulli random variable that $v_{i}$ marginally contributes $v_{j}$ to fringe $\left(C_{i}\right)$. Thus:

$$
E\left[B_{v_{i}, v_{j}}\right]=\operatorname{Pr}\left[\left(N_{G}\left(v_{j}\right) \cup\left\{v_{j}\right\}\right) \cap C_{i}=\emptyset\right]=\frac{1}{1+\operatorname{deg}_{G}\left(v_{j}\right)}
$$

Therefore, the Shapley Value $S V_{g_{1}}\left(v_{i}\right)$, which is the expected marginal contribution of $v_{i}$, is given by:

$$
S V_{g_{1}}\left(v_{i}\right)=\sum_{v_{j} \in\left\{v_{i}\right\} \cup N_{G}\left(v_{i}\right)} E\left[B_{v_{i}, v_{j}}\right]=\sum_{v_{j} \in\left\{v_{i}\right\} \cup N_{G}\left(v_{i}\right)} \frac{1}{1+\operatorname{deg} g_{G}\left(v_{j}\right)}
$$

which is an exact closed-form expression for computing the SV of each node on the network.

Algorithm 1 describes an $O(V+E)$ procedure that directly implements the above equation to compute the exact SVs of all nodes in the network. By contrast, MonteCarlo simulation requires $O(V+E)$ operations for every iteration. Moreover, the results obtained using Monte-Carlo are statistical in nature and may not be sufficiently accurate unless a large number of iterations are carried out. 


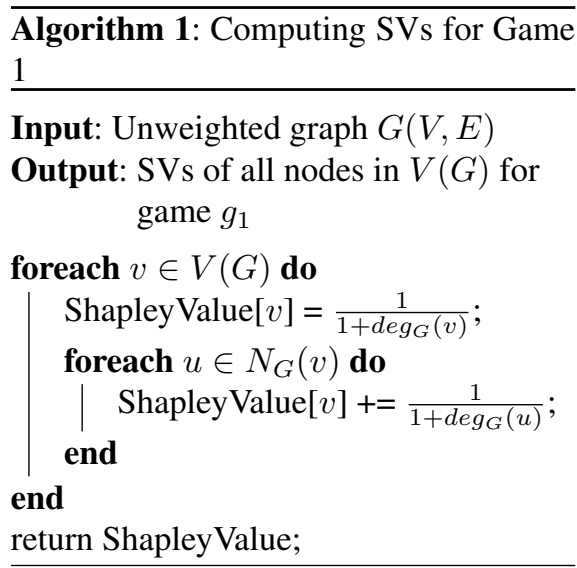

It is possible to derive some intuition from the above formula. If a node has a high degree, the number of terms in its SV summation above will also be high. But the terms themselves would be inversely related to the degree of neighboring nodes. This gives the intuition that a node will have high centrality not only when its degree is high, but also whenever its degree tends to be higher in comparison to the degree of its neighboring nodes. In other words, power comes from being connected to those who are powerless, a fact that is well-recognized [13] by the centrality literature.

\subsection{Game 2: $\nu_{2}(C)=$ \#agents with at-least $k$ neighbors in $C$}

We now consider a more general game formulation for an unweighted graph $G(V, E)$, where the value of a coalition includes the number of agents who are either in the coalition or are adjacent to at least $k$ agents who are in the coalition. Formally, we consider game $g_{2}$ characterised by $\nu_{2}: 2^{V(G)} \rightarrow \mathbb{R}$, where

$$
\nu_{2}(C)= \begin{cases}0 & \text { if } C=\emptyset \\ \mid\left\{v: v \in C \text { (or) }\left|N_{G}(v) \cap C\right| \geq k\right\} \mid & \text { else }\end{cases}
$$

Note that this game reduces to game $g_{1}$ for $k=1$.

The motivation for this generalization is that in many real-life networks, the value of a coalition is interpreted as the number of agents who can be "influenced" by the coalition. For instance, in a viral marketing or innovation diffusion analysis [14], it is usually assumed that an agent will "be influenced" only if atleast $k$ of his neighbors have already been convinced, which suggests such a game formulation.

Adopting notation from the previous subsection, we again ask: what is the necessary and sufficient condition for node $v_{i}$ to marginally contribute node $v_{j} \in N_{G}\left(v_{i}\right) \cup\left\{v_{i}\right\}$ to the value of the coalition $C_{i}$ ?

Clearly, if $\operatorname{deg}_{G}\left(v_{j}\right)<k$, we have $E\left[B_{v_{i}, v_{j}}\right]=\delta\left(v_{i}, v_{j}\right)$, i.e, $E\left[B_{v_{i}, v_{j}}\right]=1$ for $v_{i}=v_{j}$ and 0 otherwise.

For $\operatorname{deg}_{G}\left(n_{j}\right) \geq k$, we split the argument into two cases. If $v_{j} \neq v_{i}$, the condition for marginal contribution is that exactly $(k-1)$ neighbors of $v_{j}$ already belong to $C_{i}$ and $v_{j} \notin C_{i}$. On the other hand, if $v_{j}=v_{i}$, marginal contribution happens if and only if $C_{i}$ originally consisted of at most $(k-1)$ neighbors of $v_{j}$.

So for $\operatorname{deg}_{G}\left(v_{j}\right) \geq k$ and $v_{j} \neq v_{i}$, we have

$$
E\left[B_{n_{i}, n_{j}}\right]=\left(\begin{array}{c}
\operatorname{deg}_{G}\left(v_{j}\right)-1 \\
k-1
\end{array}\right) \frac{(k-1) !\left(1+\operatorname{deg}_{G}\left(v_{j}\right)-k\right) !}{\left(1+\operatorname{deg}_{G}\left(v_{j}\right)\right) !}=\frac{1+\operatorname{deg}_{G}\left(v_{j}\right)-k}{\operatorname{deg}_{G}\left(v_{j}\right)\left(1+\operatorname{deg}_{G}\left(v_{j}\right)\right)}
$$


And for $\operatorname{deg}_{G}\left(v_{i}\right) \geq k$ and $v_{j}=v_{i}$, we have

$$
E\left[B_{v_{i}, v_{i}}\right]=\frac{k}{1+\operatorname{deg}_{G}\left(v_{i}\right)}
$$

As before, the SVs are given by substituting the above formulas into:

$$
S V_{g_{2}}\left(v_{i}\right)=\sum_{v_{j} \in N_{G}\left(v_{i}\right) \cup\left\{v_{i}\right\}} E\left[B_{v_{i}, v_{j}}\right]
$$

Although this game is a generalization of game $g_{1}$, it can still be solved to obtain the SVs of all nodes in $O(V+E)$ time, as formalised by Algorithm 2 .

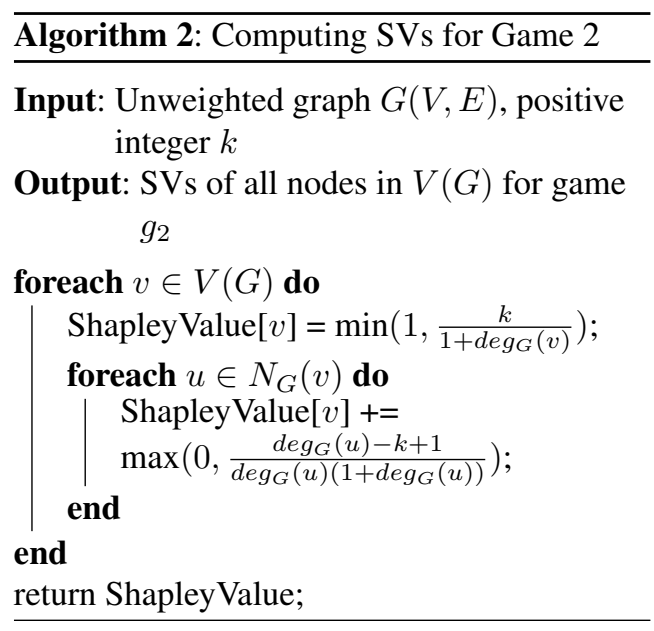
An even more general formulation of the game is possible by allowing $k$ to be a function of the agent, i.e, each node $v_{i} \in V(G)$ is assigned its own unique attribute $k\left(v_{i}\right)$. This translates to an application of the form: agent $i$ is convinced if and only if atleast $k_{i}$ of his neighbors are convinced, which is a frequently used model in the literature [14].

The above proof does not use the fact that $k$ is constant across all nodes. So this generalized formulation can be solved by a simple modification to the original SV expression:

$$
S V\left(v_{i}\right)=\frac{k\left(v_{i}\right)}{1+\operatorname{deg}_{G}\left(v_{i}\right)}+\sum_{v_{j} \in N_{G}\left(v_{i}\right)} \frac{1+\operatorname{deg}_{G}\left(v_{j}\right)-k\left(v_{j}\right)}{\operatorname{deg}_{G}\left(v_{j}\right)\left(1+\operatorname{deg}_{G}\left(v_{j}\right)\right)}
$$

The above equation (which is also implementable in $O(V+E)$ time) assumes that $k\left(v_{i}\right) \leq 1+\operatorname{deg}_{G}\left(v_{i}\right)$ for all nodes $v_{i}$. This condition can be assumed without loss of generality because all cases can still be modeled (we set $k\left(v_{i}\right)=1+\operatorname{deg}_{G}\left(v_{i}\right)$ for the extreme case where node $v_{i}$ is never convinced no matter how many of its neighbors are already convinced).

\subsection{Game 3: $\nu_{3}(C)=$ \#agents at-most $d_{\text {cutoff }}$ away}

Hitherto, our games have been confined to unweighted networks. But in many applications, it is necessary to model real-world networks as weighted graphs. For example, in a co-authorship network, each edge is often assigned a weight proportional to the number of joint publications the corresponding authors have produced [15].

This subsection extends the game $g_{1}$ to the case of weighted networks. Whereas game $g_{1}$ equates $\nu(C)$ to the number of nodes located within one hop of some node in $C$, our new formulation in this subsection equates $\nu(C)$ to the number of nodes located within a distance $d_{\text {cutoff }}$ of some node in $C$. Here, distance between two nodes is measured as the length of the shortest path between the nodes in the given weighted graph $G(V, E, W)$, where $W: E \rightarrow \mathbb{R}^{+}$is the weight function.

Formally, we define the game $g_{3}$, where for each coalition $C \subseteq V(G)$,

$$
\nu_{3}(C)= \begin{cases}0 & \text { if } C=\emptyset \\ \operatorname{size}\left(\left\{v_{i}: \exists v_{j} \in C \mid \operatorname{distance}\left(v_{i}, v_{j}\right) \leq d_{\text {cutoff }}\right\}\right) & \text { else }\end{cases}
$$


We shall now show that even this highly general centrality game $g_{3}$ is amenable to analysis which yields an exact formula for SVs. However, in this case the algorithm for implementing the formula is not linear in the size of the network, but has $O(V E+$ $\left.V^{2} \log (V)\right)$ complexity.

Let us introduce some extra notation. Define the extended neighborhood $N_{G}\left(v_{j}, d_{\text {cutoff }}\right)=$ $\left\{v_{k} \neq v_{j}\right.$ : distance $\left.\left(v_{k}, v_{j}\right) \leq d_{\text {cutoff }}\right\}$, i.e, the set of all nodes whose distance from $v_{j}$ is at most $d_{\text {cutoff. }}$ Denote the size of $N_{G}\left(v_{j}, d_{\text {cutoff }}\right)$ by $\operatorname{deg}_{G}\left(v_{j}, d_{\text {cutoff }}\right)$.

With this notation, the necessary and sufficient condition for node $v_{i}$ to marginally contribute node $v_{j}$ to the value of coalition $C_{i}$ is: distance $\left(v_{i}, v_{j}\right) \leq d_{\text {cutoff }}$ and distance $\left(v_{j}, v_{k}\right)>$ $d_{\text {cutoff }} \forall v_{k} \in C_{i}$. That is, neither $v_{j}$ nor any node in its extended neighborhood should be present in $C_{i}$. But from the discussion of previous subsections, we know that the probability of this event is exactly $\frac{1}{1+\operatorname{deg}_{G}\left(v_{j}, d_{\text {cutoff }}\right)}$. Therefore, the exact formula for SV of node $v_{i}$ in game $g_{3}$ is:

$$
S V_{g_{3}}\left(v_{i}\right)=\sum_{v_{j} \in\left\{v_{i}\right\} \cup N_{G}\left(v_{i}, d_{\text {cutoff }}\right)} \frac{1}{1+\operatorname{deg}_{G}\left(v_{j}, d_{\text {cutoff }}\right)}
$$

Algorithm 3 works as follows: for each node $v$ in the network $G(V, E)$, the extended neighborgood $N_{G}\left(v, d_{\text {cutoff }}\right)$ and its size $\operatorname{deg}_{G}\left(v, d_{\text {cutoff }}\right)$ are first computed using Dijkstra's algorithm in $O(E+V \log (V))$ time [16]. The results are then used to directly implement the above equation, which takes maximum time $O\left(V^{2}\right)$. In practice this step runs much faster because the worst case situation only occurs when every node is reachable from every other node within $d_{\text {cutoff }}$ Overall the complexity of the algorithm is $O\left(V E+V^{2} \log (V)\right)$.

We make one final observation: that the above proof does not depend on $d_{\text {cutoff }}$ being constant across all nodes. Indeed, each node $v_{i} \in V(G)$ may be assigned its own unique value $d_{\text {cutoff }}\left(v_{i}\right)$, where $\nu(C)$ would be the number of agents $v_{i}$ who are within a distance $d_{\text {cutoff }}\left(v_{i}\right)$ from $C$. For this case, the above proof gives:

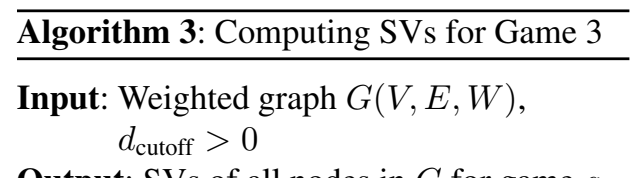

Output: SVs of all nodes in $G$ for game $g_{3}$

foreach $v \in V(G)$ do

DistanceVector D = Dijkstra $(v, G)$;

$\operatorname{extNeighbors}(v)=\emptyset$; $\operatorname{extDegree}(v)=0$;

foreach $u \in V(G)$ such that $u \neq v$ do if $D(u) \leq d_{\text {cutoff }}$ then extNeighbors $(v) \cdot \operatorname{push}(u)$; end $\operatorname{extDegree}(v)++$;

end

end

foreach $v \in V(G)$ do

ShapleyValue $[v]=\frac{1}{1+\operatorname{extDegree}(v)}$;

foreach $u \in \operatorname{extNeighbors}(v)$ do

ShapleyValue $[v]+=$

$\frac{1}{1+\text { extDegree }(u)}$

end

end

return ShapleyValue;

$$
S V\left(v_{i}\right)=\sum_{\substack{v_{j}: \operatorname{distance}\left(v_{i}, v_{j}\right) \\ \leq d_{\text {cutoff }}\left(v_{j}\right)}} \frac{1}{1+\operatorname{deg}_{G}\left(v_{j}, d_{\text {cutoff }}\left(v_{j}\right)\right)}
$$


3.4 Game 4: $\nu_{4}(C)=\sum_{v_{i} \in V(G)} f\left(\operatorname{distance}\left(v_{i}, C\right)\right)$

This subsection further generalizes game $g_{3}$, again taking motivation from real-life network problems. In game $g_{3}$, all agents at distances $d_{\text {agent }} \leq d_{\text {cutoff }}$ contributed equally to the value of a coalition. However, this assumption may not always hold true because in some applications, we intuitively expect agents closer to a coalition to contribute more to its value. For instance, we expect a Facebook user to exert more influence over his immediate circle of friends than over "friends of friends", even though both may satisfy the $d_{\text {cutoff }}$ criterion. Similarly, we expect a virus-affected computer to infect a neighboring computer more quickly than a computer two hops away.

In general, we expect that an agent at distance $d$ from a coalition would contribute $f(d)$ to its value, where $f($.$) is a positive valued decreasing function of its argument. More$ formally, we define the game $g_{4}$ where the value of a coalition $C$ is given by:

$$
\nu_{4}(C)= \begin{cases}0 & \text { if } C=\emptyset \\ \sum_{v_{i} \in V(G)} f\left(d\left(v_{i}, C\right)\right) & \text { else }\end{cases}
$$

where $d\left(v_{i}, C\right)$ is the minimum distance $\min \left\{\operatorname{distance}\left(v_{i}, v_{j}\right) \mid v_{j} \in C\right\}$.

It is possible to solve for SVs in the above formulation by constructing a marginal contribution network (MC-Net) [7]. However, the MC-Net so constructed would have $O\left(V^{3}\right)$ rules. In the discussion below, we give a more efficient algorithm that runs in $O\left(V E+V^{2} \log (V)\right)$. This is a considerable improvement because most real-world networks for which this formulation computes centralities are sparse, i.e, $E \sim O(V)$.

The key question to ask is: what is the expected value of the marginal contribution of $v_{i}$ through node $v_{j} \neq v_{i}$ to the value of coalition $C_{i}$ ? Let this marginal contribution be denoted $M C\left(v_{i}, v_{j}\right)$. Clearly:

$$
M C\left(v_{i}, v_{j}\right)= \begin{cases}0 & \text { if distance }\left(v_{i}, v_{j}\right) \geq d\left(v_{j}, C_{i}\right) \\ f\left(\operatorname{distance}\left(v_{i}, v_{j}\right)\right)-f\left(d\left(v_{j}, C_{i}\right)\right) & \text { else }\end{cases}
$$

Let $D_{v_{j}}=\left\{d_{1}, d_{2} \ldots d_{|V|-1}\right\}$ be the distances of node $v_{j}$ from all other nodes in the network, sorted in increasing order. Let the nodes corresponding to these distances be $\left\{w_{1}, w_{2} \ldots w_{|V|-1}\right\}$ respectively. Let $k_{i j}+1$ be the number of nodes (out of these $|V|-1$ ) whose distances to $v_{j}$ are $\leq \operatorname{distance}\left(v_{i}, v_{j}\right)$. Let $w_{k_{i j}+1}=v_{i}$ (i.e, among all nodes that have the same distance from $v_{j}$ as $v_{i}, v_{i}$ is placed last in the increasing order).

We use literal $w_{i}$ to mean $w_{i} \in C_{i}$ and the literal $\overline{w_{i}}$ to mean $w_{i} \notin C_{i}$. Define a sequence of boolean variables $p_{k}=\overline{v_{j}} \wedge \overline{w_{1}} \wedge \overline{w_{2}} \wedge \ldots \wedge \overline{w_{k}}$ for each $0 \leq k \leq|V|-1$. Finally denote expressions of the form $M C\left(v_{i}, v_{j} \mid F\right)$ to mean the marginal contribution of $v_{i}$ to $C_{i}$ through $v_{j}$ given that the coalition $C_{i}$ satisfies the boolean expression $F$.

$$
\begin{gathered}
M C\left(v_{i}, v_{j} \mid p_{k_{i j}+1} \wedge w_{k_{i j}+2}\right)=f\left(d_{k_{i j}+1}\right)-f\left(d_{k_{i j}+2}\right) \\
M C\left(v_{i}, v_{j} \mid p_{k_{i j}+2} \wedge w_{k_{i j}+3}\right)=f\left(d_{k_{i j}+1}\right)-f\left(d_{k_{i j}+3}\right) \\
\vdots \quad \vdots \quad \vdots \\
M C\left(v_{i}, v_{j} \mid p_{|V|-2} \wedge w_{|V|-1}\right)=f\left(d_{k_{i j}+1}\right)-f\left(d_{|V|-1}\right) \\
M C\left(v_{i}, v_{j} \mid p_{|V|-1}\right)=f\left(d_{k_{i j}+1}\right)
\end{gathered}
$$


With this notation, we obtain expressions for $M C\left(v_{i}, v_{j}\right)$ by splitting over the above mutually exclusive and exhaustive (i.e, covering all possible non-zero marginal contributions) cases.

The probabilities $\operatorname{Pr}\left(p_{k} \wedge w_{k+1}\right)$ are found by elementary combinatorics which gives:

$$
\operatorname{Pr}\left(p_{k} \wedge w_{k+1}\right)=\frac{k !}{(k+2) !}=\frac{1}{(k+1)(k+2)} \forall 1+k_{i j} \leq k \leq|V|-2
$$

Using the $M C\left(v_{i}, v_{j}\right)$ equations and the probabilities $\operatorname{Pr}\left(p_{k} \wedge w_{k+1}\right)$ :

$$
\begin{aligned}
E\left[M C\left(v_{i}, v_{j}\right)\right] & =\left[\sum_{k=1+k_{i j}}^{|V|-2} \frac{f\left(\operatorname{distance}\left(v_{i}, v_{j}\right)\right)-f\left(d_{k+1}\right)}{(k+1)(k+2)}\right]+\frac{f\left(\operatorname{distance}\left(v_{i}, v_{j}\right)\right)}{|V|} \\
& =\frac{f\left(\operatorname{distance}\left(v_{i}, v_{j}\right)\right)}{k_{i j}+2}-\sum_{k=k_{i j}+1}^{|V|-2} \frac{f\left(d_{k+1}\right)}{(k+1)(k+2)}
\end{aligned}
$$

For $v_{i}=v_{j}$, a similar analysis produces:

$$
E\left[M C\left(v_{i}, v_{i}\right)\right]=f(0)-\sum_{k=0}^{|V|-2} \frac{f\left(d_{k+1}\right)}{(k+1)(k+2)}
$$

Finally the exact SVs are given by:

$S V_{g_{4}}\left(v_{i}\right)=\sum_{v_{j} \in V(G)} E\left[M C\left(v_{i}, v_{j}\right)\right]$

Algorithm 4 implements the above formulas. For each vertex $v$, a vector of distances to every other vertex is first computed using Dijkstra's algorithm [16]. This yields a vector $D_{v}$ that is already sorted in increasing order. This vector is then traversed in reverse, to compute the backwards cumulative sum $\sum \frac{f\left(d_{k+1}\right)}{(k+1)(k+2)}$. At each step of the backward traversal, the SV of the appropriate node $w$ is updated according to the $E[M C(w, v)]$ equation. After the traversal, the SV of $v$ itself is updated according to the $E[M C(v, v)]$ equation. This process is repeated for all nodes $v$ so that at the end of the algorithm, all SVs have been computed exactly in $O\left(V E+V^{2} \log (V)\right)$ time.

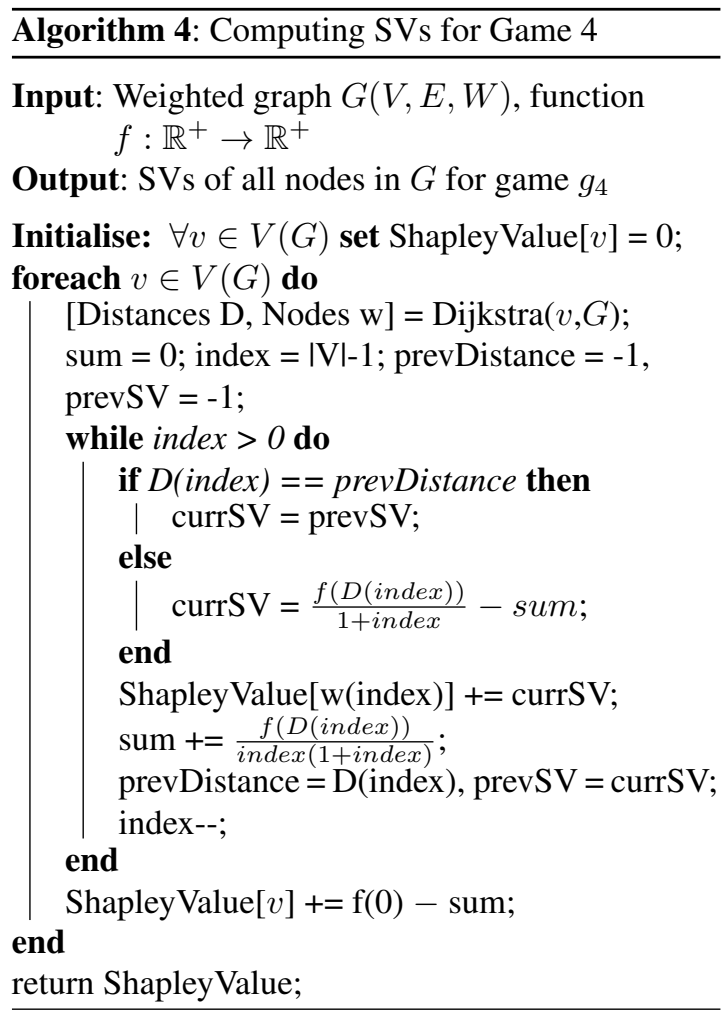




\section{Summary and conclusions}

\begin{tabular}{|c|c|c|c|}
\hline Game & Graph & $\nu(C)$ & Complexity \\
\hline \hline$g_{1}$ & $U W$ & $\leq 1$ degree away & $V+E$ \\
\hline$g_{2}$ & $U W$ & $\geq k$ neighbors $\in C$ & $V+E$ \\
\hline$g_{3}$ & $W$ & $\leq d_{\text {cutoff }}$ away & $V E+V^{2} \log V$ \\
\hline$g_{4}$ & $W$ & $\sum_{v_{i}} f\left(d\left(v_{i}, C\right)\right)$ & $V E+V^{2} \log V$ \\
\hline
\end{tabular}

The table to the left presents a brief summary of the SV algorithms discussed in this paper. These algorithms enable efficient centrality computation for many real-world applications

$\{W=$ weighted, $U W=$ unweighted $\}$ including the analysis of social netof epidemics, biological and biochemical networks, viral marketing and internet/web phenomena.

The conclusion is that many centrality-related co-operative games of interest played on real-life networks can in fact be solved for SVs analytically. The resulting algorithms are not only error-free but also run in polynomial time and in practice, much faster than Monte-Carlo methods. Approximate closed-form expressions and algorithms can also be constructed for some classes of games played on weighted networks. Simulation results (please see on-line appendix) show that these approximations are quite acceptable.

\section{References}

1. N.R. Suri and Y. Narahari. Determining the top-k nodes in social networks using the Shapley Value. In AAMAS '08: Proceedings of the Seventh International Joint Conference on Autonomous Agents and Multi-Agent Systems, pages 1509-1512, 2008.

2. D. Gómez, E. González-Aranguiena, C. Manuel, G. Owen, M. Del Pozo, and J. Tejada. Centrality and power in social networks: A game theoretic approach. Mathematical Social Sciences, 46(1):27-54, 2003.

3. D.J. Watts. Six degrees: The science of a connected age. W.W. Norton \& Company, 2004.

4. D. Kempe, J. Kleinberg, and É. Tardos. Influential nodes in a diffusion model for social networks. Automata, Languages and Programming, 2005.

5. N.R. Suri and Y. Narahari. A Shapley Value based approach to discover influential nodes in social networks. IEEE Trans. on Automation Science and Engineering, 99:1-18, 2010.

6. X. Deng and C. Papadimitriou. On the complexity of cooperative solution concepts. Mathematics of Operations Research, 19(2):257-266, 1994.

7. S. Ieong and Y. Shoham. Marginal contribution nets: a compact representation scheme for coalitional games. In EC '05: Proceedings of the Sixth ACM Conference on Electronic Commerce, pages 193-202, 2005.

8. D. Koschützki, K.A. Lehmann, L. Peeters, S. Richter, D. Tenfelde-Podehl, and O. Zlotowski. Centrality indices. Network analysis, volume 3418 of Lecture Notes in Computer Science, pages 16-61. Springer, 2005.

9. J.A. Bondy and U.S.R. Murty. Graph theory and its applications. Macmillan, 1976.

10. D.B. West. Introduction to graph theory. Prentice Hall, Inc., 2001.

11. R. Gibbons. A primer in game theory. Harvester Wheatsheaf, 1994.

12. A. Rapoport. N-person game theory: Concepts and applications. Dover Publications, 2001.

13. P. Bonacich. Power and centrality: A family of measures. American Journal of Sociology, 92(5):1170-1182, 1987.

14. T.W. Valente. Social network thresholds in the diffusion of innovations. Social Networks, 18(1):69-89, 1996.

15. M.E.J. Newman. Scientific collaboration networks. II. Shortest paths, weighted networks, and centrality. Physical Review E, 64(1):016132(1-7), 2001.

16. T.H. Cormen. Introduction to algorithms. MIT Press, 2001. 